\title{
Orcein-alcian blue staining: a new technique for demonstrating acid mucins in gastrointestinal epithelium
}

\author{
R SINGH, A W P GORTON Department of Histopathology, Manor Hospital, Walsall, West Midlands
}

SUMMARY Orcein-alcian blue staining, a new method for the simultaneous demonstration of sulphated and sialomucins in gastrointestinal epithelium was compared with the standard high iron diamine-alcian blue technique. Sections were oxidised with potassium permanagate and decolourised in oxalic acid. They were stained with orcein for four hours, differentiated for a few seconds in acid alcohol, and then counterstained with alcian blue for half to one minute. There was a good correlation of results between the two methods. Orcein-alcian blue is a safer, cheaper, and quicker method than high iron diamine-alcian blue which can be safely introduced into routine laboratories for the study of acid mucins in the gastrointestinal diseases.

Orcein, a naturally occurring vegetable dye, has been used for staining elastic fibres for many years. It is also used for the demonstration of hepatitis B antigen and copper-associated proteins in chronic liver diseases. ${ }^{2}$

Recently, the orcein technique has been studied for showing the presence of sulphated mucin in gastrointestinal epithelium. ${ }^{34}$ It has also been reported to be selectively positive for mucin-producing adenocarcinomas of the lower gastrointestinal tract; therefore, adenocarcinomas in sites other than the colon and rectum are negative for orcein staining. ${ }^{5}$ We report our findings of combining a modified orcein staining method with alcian blue to show the presence of sulphated and sialomucins in normal, non-neoplastic, and neoplastic gastrointestinal epithelium.

\section{Material and methods}

The details of the 47 cases studied are shown in the table. The resected surgical specimens were fixed in $10 \%$ formol-saline and routinely processed for paraffin wax embedding. Serial sections, $5 \mu \mathrm{m}$ thick, were stained with haematoxylin and eosin, orcein, orceinalcian blue, high iron-diamine and high iron-diaminealcian blue (modifications of Spicer's method). ${ }^{6}$

Shikata's orcein method was used with a slight modification. ' The sections were taken to water, then oxidised in a solution of $0.25 \%$ potassium permanagate in $\mathbf{0 . 2 5 \%}$ concentrated sulphuric acid for one minute. Sections were decolourised in $2 \%$ oxalic acid and rinsed in water. They were then stained in orcein solution for four hours. This staining solution was prepared by dissolving $1.0 \mathrm{~g}$ natural orcein (Gurr, Accepted for publication 2 February 1989
Table

\begin{tabular}{lr} 
Histological diagnosis & No of cases \\
\hline Normal mucosa (stomach, ileum, appendix, large bowel) & 25 \\
Intestinal metaplasia of stomach & 5 \\
Adenomas (stomach, appendix, large bowel) & 6 \\
Adenocarcinomas (stomach, appendix, ileum, large bowel) & 11 \\
Total & 47 \\
\hline
\end{tabular}

BDH Poole, Dorset) in $100 \mathrm{ml} 70 \%$ alcohol and then adding $1.0 \mathrm{ml}$ concentrated hydrochloric acid. Sections were washed well in tap water and differentiated for a few seconds in acid-alcohol. Sections were again washed in water and stained in $1 \%$ alcian blue in $3 \%$ acetic acid ( $\mathrm{pH} 2.5)$ for one minute. This was followed by dehydration, clearing and mounting.

\section{CONTROL STUDY}

To establish that orcein stained sulphomucins in the goblet cells and not sulphur-containing amino acids, control sections of keratinised skin, normal stomach, colon and rectum were subjected to a two stage blocking technique before orcein-alcian blue staining, using sodium dithionite and mercuric chloride which effectively block any S-S and S-H groups which might be present. The presence of such sulphur-containing amino acids can then be determined by comparison with untreated, stained duplicate sections (HC Cook, personal communication).

\section{Results}

Throughout the entire gastrointestinal tract, the 


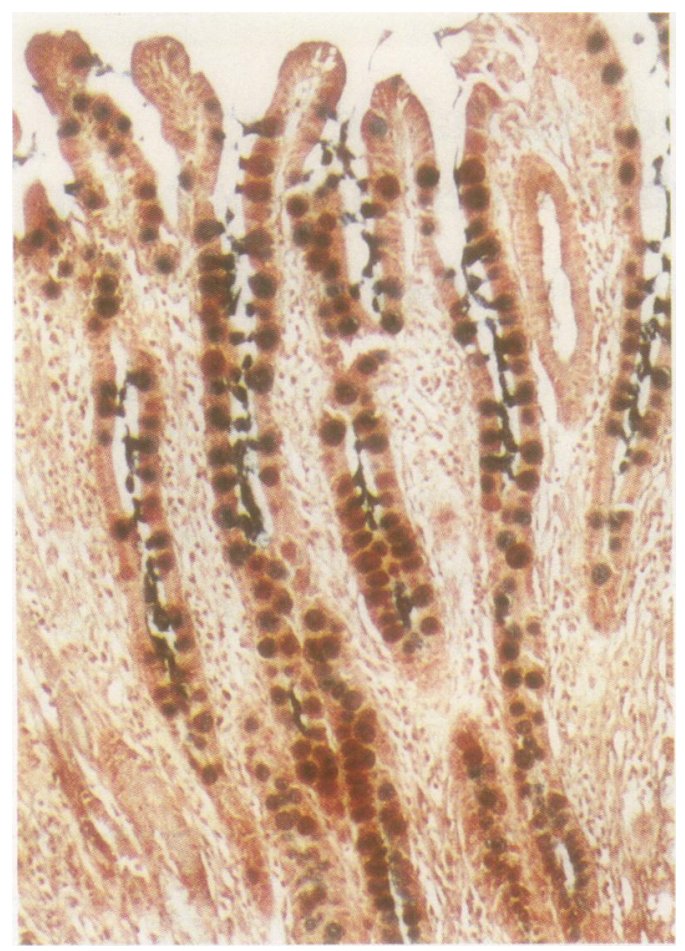

Fig 1 Section showing goblet cells of stomach intestinal metaplasia containing sulphated mucin (brown) and sialomucin (blue). (Orcein-alcian blue.)

goblet cells containing sulphated mucins stained brown with orcein and sialomucins stained blue with alcian blue. With the exception of the elastic fibres, all other cells and tissues showed a faint brown staining that was regarded as negative in this study. To compare the staining results of orcein-alcian blue and high iron diamine-alcian blue identical areas were studied in serial sections.

Surface foveolar epithelial cells of the stomach did not stain. Apical granules of the parietal cells of stomach showed a faint brown staining with orceinalcian blue. There was comparable negative staining of cells with high iron diamine-alcian blue. The goblet cells in gastric intestinal metaplasia-complete and incomplete type-however, stained for both sulphated and sialomucins (fig 1). High iron diamine-alcian blue also showed similar results. In the terminal ileum, close to the ileocaecal valve, traces of sulphomucins were seen in occasional goblet cells (fig 2). In the left colon sulphomucins were seen in the goblet cells in the lower half of the crypt and sialomucins in the upper crypt (figs $3 a$ and $b$ ); a reverse pattern was observed in the right colon and the appendix. The above results

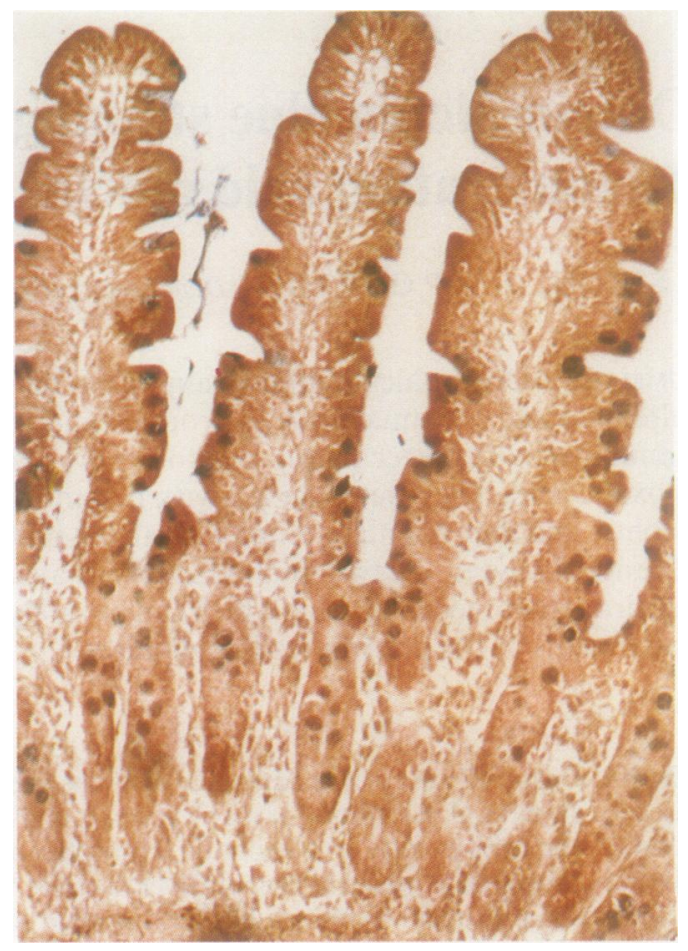

Fig 2 Section of terminal ileum showing ocassional goblet cells in the villi and crypts containing sulphomucin (brown). (Orcein-alcian blue.)

were similar to that obtained with high iron diaminealcian blue.

The six benign and 11 malignant tumours stained with orcein-alcian blue and high iron diamine-alcian blue had identical results. The benign tumours showed a production of mixed mucins with predominance of either sulphated or sialomucins. The malignant tumours varied from well differentiated mucin-secreting to poorly differentiated with a corresponding decrease in mucin content. The secretory product in all the adenocarcinomas was a mixture of mucins with predominance of either sulphated or sialomucin. The poorly differentiated adenocarcinomas showed scanty mucin in occasional goblet cells and in the lumen of gland-like structures. In mucoid carcinomas with extracellular mucin "lakes", however, there was a predominance of intra- and extracellular sialomucin and little sulphomucin production. The sections treated with dithionite stained with orcein-alcian blue showed undiminished staining in the goblet cells. The specificity and the intensity of staining with orcein was similar to that seen in untreated sections stained with orcein-alcian blue. 

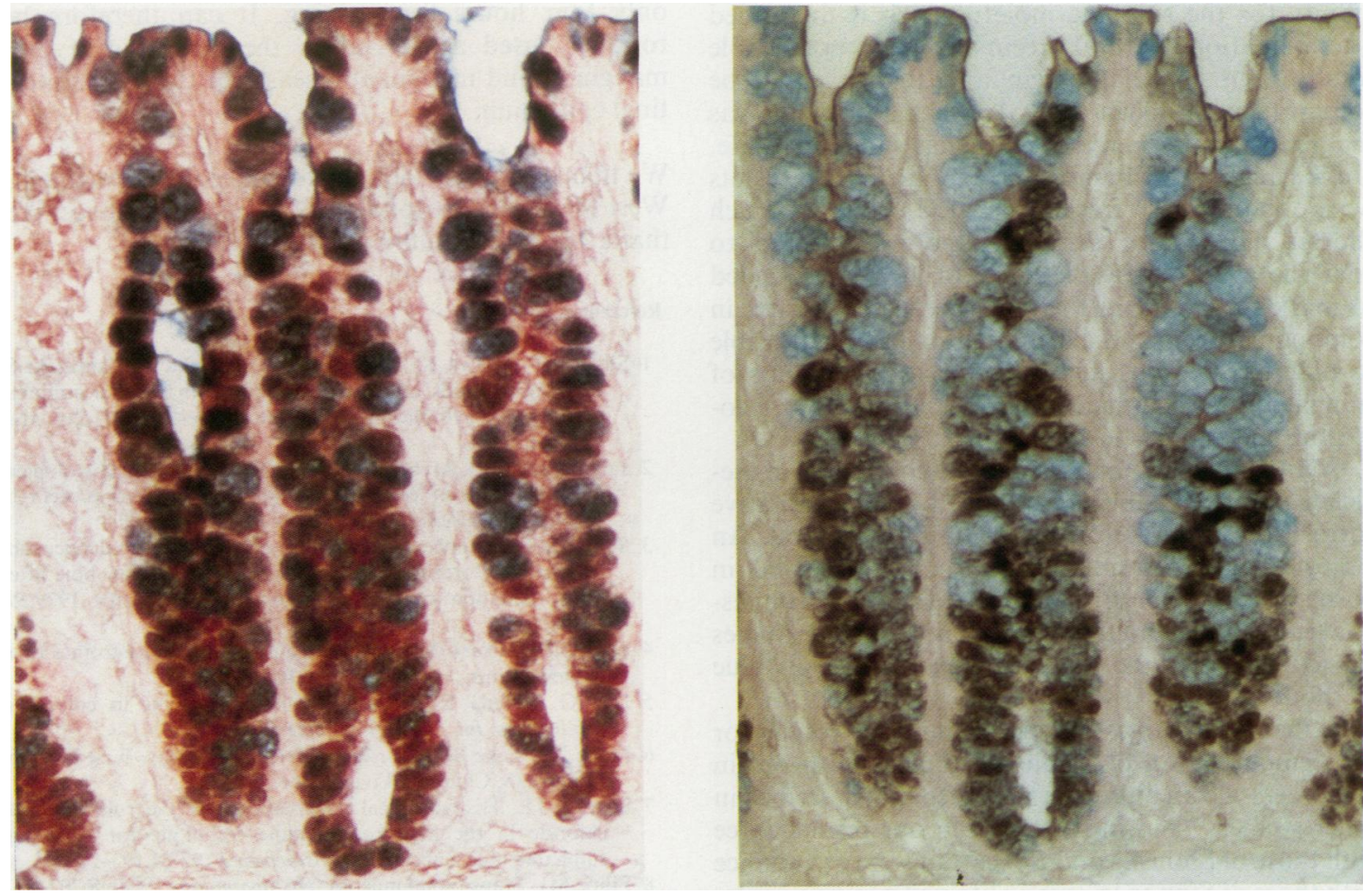

Fig 3 Normal mucosa of left colon showing predominance of sulphated mucin (brown) in lower crypt and sialomucin (blue) in the upper third of crypt: (a) orcein-alcian blue; (b) high iron diamine-alcian blue.

\section{Discussion}

Most research work carried out to study mucin secretion of gastrointestinal epithelium has been based on only one technique - that is, the high iron diaminealcian blue method. ${ }^{6}$ This stains sulphated mucins black and sialomucins blue. This technique has two fundamental disadvantages, however: (i) The diamine reagents $(\mathrm{N}, \mathrm{N}$-dimethyl-m-phenylene-diamine and $\mathrm{N}$, $\mathbf{N}$ dimethyl-p-phenylenediamine) are carcinogenic and hence require careful handling; (ii) it requires at least an 18 hour staining period. The other drawbacks of this method are that some of the steps are crucial for correct results. These include the commercial source of the reagents, the water rinse, $\mathrm{pH}$ of the solutions, concentration of the ferric chloride solution, and the use of freshly prepared diamine salts. Above all, the variations in staining method have also been reported as giving different results. ${ }^{6-8}$

Recently, the KOH-AB1-0-PAPS method ${ }^{9}$ has been used for detecting sulphated and sialomucins in the gastrointesinal epithelium and the results were compared with high iron diamine-alcian blue. It is claimed that this technique is more sensitive in detecting cells containing both types of mucins. Reproducibility of this method, however, has yet to be investigated in other routine laboratories, and the lengthy staining time (more than 24 hours) and the relative complexity may deter laboratories from using it routinely.

Sipponen studied the distribution of cells positive for orcein in the gastrointestinal tract and compared the results with high iron diamine and AB $1 \cdot 0$ and $2 \cdot 5 .^{3}$ With or without prior oxidation orcein, high iron diamine and $A B 1.0$ gave similar results: it was concluded that, like high iron diamine or AB 1.0, the orcein reactions stain sulphate groups in epithelial mucosubstances and that sulphonic acid residues, resulting from the oxidation of sulphur groups (S-S or $\mathrm{S}-\mathrm{H}$ ) in the protein core of mucus glycoproteins were responsible for the orcein, high iron diamine and alican blue reactions. The application of the dithionite blocking technique, however, which gave an unchanged orcein staining effect, meant that we were able to show that orcein was staining sulphated mucin only in the material studied in spite of the oxidation step.

In this study we found that the intensity of orcein staining was stronger with oxidation than without. This can be explained by the fact that potassium permanganate oxidation enhances polyanionic reactions and also that orcein has a natural selective 
affinity for the sulphate moiety which is intensified after oxidation. Natural orcein also gave more stable results than artificial orcein and that it could be combined with alican-blue. Another advantage was that prepared orcein matures and has a long shelf-life. Unlike high iron diamine-alcian blue, poor results were obtained with freshly prepared orcein. A much shorter alcian blue staining time was needed to combine with orcein as the standard 30 minutes tended to mask the orcein in the goblet cells. Therefore, in combining orcein and alcian blue stains, it is possible to differentiate consistently the relative amounts of sulphated and sialomucins in the normal, non-neoplastic, and neoplastic epithelium.

Compared with the standard high iron diaminealcian blue technique, we obtained good qualitative correlation using the orcein-alcian blue method. An almost identical staining pattern was observed in normal, non-neoplastic, and neoplastic gastrointestinal epithelium, and this has been described in studies carried out with the high iron diamine-alcian blue method. ${ }^{10}$

In the normal intestinal epithelium our results for the demonstration of sulphated mucins with orcein and orcein-alcian blue were similar to that described in other studies, ${ }^{34}$ but in the stomach our results agree with those of Shousha and Boxer ${ }^{4}$ but were at variance with those of Sipponen, ${ }^{3}$ who obtained positive oxidation-orcein staining of surface foveolar cells. Sipponen's results are surprising in view of the accepted lack of polyanions in normal human gastric epithelium.

Taking into consideration the problems discussed with the existing high iron diamine-alcian blue and $\mathrm{KOH}-\mathrm{AB1} \cdot 0-\mathrm{PAPS}$ methods, orcein-alcian blue is an alternative, safer, and inexpensive method requiring only four hours for staining. It can therefore be routinely used as an aid to the diagnosis of premalignant and malignant diseases of the gastrointestinal epithelium.

We thank Mr HC Cook, histopathology department, West Middlesex Hospital, for the technical advice. We thank Mr JD Hamilton for the photographs.

\section{References}

1 Shikata T, Uzawa T, Yoshiwara N, Akatsuka T, Yamazaki S. Staining methods of Australian antigen in paraffin sectionsdetection of cytoplasmic inclusions bodies. Jpn J Exp Med 1974; $44: 25-36$

2 Jain S, Scheur PJ, Archer B, Newman SP, Sherlock S. Histological demonstration of copper and copper associated protein in chronic liver disease. J Clin Pathol 1978;31:784-90.

3 Sipponen P. Histochemical reactions of gastrointestinal mucosubstances with orcein, high iron-diamine and alcian blue after prior oxidation of tissue sections. Histochemistry 1979;59: 199-206.

4 Shousha S, Boxer GM. Orcein as a mucin stain for gastrointestinal tissue sections. J Clin Pathol 81;34:1063-4.

5 Dev G, Saxena R. Orcein-A stain for mucins in colorectal carcinomas. Int J Cancer 1984;21:68-70.

6 Filipe MI, Lake BD. Histochemistry in Pathology. First Edition. Edinburgh: Churchill Livingstone, 1983.

7 Sorvari TE. Histochemical observations on the role of ferric chloride in the high-iron diamine technique for localizing sulphated mucosubstances. Histochem $J$ 1972;4:193-204.

8 Filipe MI. Transitional mucosa. Histopathology 1984b;8:707-8.

9 McFadden DE, Owen DA, Reid PE, Jones EA. The histochemical assessment of sulphated and non-sulphated sialomucins in intestinal epithelium. Histopathology 1985;9:1129-37.

10 Filipe MI. Mucins in the human gastrointestinal epithelium: a review. Invest Cell Pathol 1979;2:195-216.

Requests for reprints to: Dr $\mathbf{R}$ Singh, Department of Histopathology, Manor Hospital, Moat Road, Walsall WS2 9PS, West Midlands. 